discriminant analysis, the sensitivity was $88.0 \%$, specificity was $67.9 \%$, accuracy was $80.8 \%$, and ROC-AUC was $0.91(95 \%$ CI: 0.85 to 0.97$)$ in the training set. In the validation set, the sensitivity was $66.7 \%$, specificity was $71.4 \%$, accuracy was $70.0 \%$, and ROC-AUC was 0.86 (95\% CI: 0.69 to 1.00 ).

Discussion Breath test may have potential in screening for pneumoconiosis. A multi-centre study is warranted to establish a reliable model and the procedures must be standardised to prevent confounding factors before clinical application.

\section{ASBESTOSIS AND GENE-ENVIRONMENT INTERACTIONS}

${ }^{1} \mathrm{~A}$ Franko, ${ }^{2} \mathrm{~V}$ Dolzan, ${ }^{1} \mathrm{M}$ Dodic-Fikfak. ${ }^{1}$ Clinical Institute of Occupational Medicine, University Medical Centre, Ljubljana, Slovenia; ${ }^{2}$ Pharmacogenetics Laboratory, Institute of Biochemistry, Faculty of Medicine, University of Ljubljana, Slovenia

\subsection{6/oemed-2018-ICOHabstracts. 1308}

Introduction Although the causal relationship between asbestosis and asbestos exposure has been well proved, little is known about the genetic factors that may influence the development of this disease. This study investigated the influence of gene-gene and geneenvironment interactions on the risk of developing asbestosis.

Methods The nested case-control study included 262 cases with asbestosis and 265 controls with no asbestos-related disease studied for MnSOD, ECSOD, CAT, GSTT1, GSTM1, GSTP1, and iNOS polymorphisms. Data on cumulative asbestos exposure and smoking were available for all subjects. PCR based methods were used for genotyping. Logistic regression analysis was used to asses asbestosis risk.

Result The OR of asbestosis was 3.21 (95\% CI: 2.43 to 4.23 ) for cumulative asbestos exposure; 0.98 (95\% CI: 0.69 to 1.39) for smoking; 1.50 (95\% CI: 1.01 to 2.24) for $M n S O D$ -9Ala/Ala versus $\mathrm{Ala} / \mathrm{Val}$ and $\mathrm{Val} / \mathrm{Val} ; 1.63$ (95\% CI: 0.62 to 4.27) for ECSOD 213Arg/Gly versus Arg/Arg; 1.36 (95\% CI: 0.70 to 2.62$)$ for CAT -262TT versus CT and CC; 1.20 (95\% CI: 0.85 to 1.69 ) for $i$ NOS LL versus SL and SS; 1.01 (95\% CI: 0.71 to 1.43 ) for GSTM1-null; 0.61 (95\% CI: 0.40 to 0.94 ) for GSTT1-null; 1.52 (95\% CI: 1.08 to 2.15 ) for GSTP1 105Ile/Ile versus 105Ile/Val and 105 Val/Val; and 0.97 (95\% CI: 0.64 to 1.48 ) for GSTP1 114Ala/Ala versus 114Ala/ $\mathrm{Val}$ and $114 \mathrm{Val} / \mathrm{Val}$. The associations between MnSOD Ala9 Val polymorphism and asbestosis, and between iNOS genotypes and asbestosis $(\mathrm{p}=0.038)$ were modified by CAT$262 \mathrm{C}>\mathrm{T}$ polymorphism $(\mathrm{p}=0.031)$. A strong interaction was found between GSTM1-null polymorphism and smoking $(\mathrm{p}=0.007), \quad$ iNOS $(\text { CCTTT })_{\mathrm{n}}$ polymorphism and smoking $(\mathrm{p}=0.054)$ as well as between $i$ NOS $(\text { CCTTT })_{n}$ polymorphism and cumulative asbestos exposure $(\mathrm{p}=0.037)$.

Discussion The findings suggest that in addition to asbestos exposure the interactions between different genotypes, genotypes and smoking, and between genotypes and asbestos exposure have an important influence on developing asbestosis and should be considered seriously in future research on occupational/environmental asbestos-related diseases.

\section{2}

SPIROMETRY LONGITUDINAL DATA ANALYSIS SOFTWARE (SPIROLA) FOR RESPIRATORY DISEASE PREVENTION AMONG SHIPYARD WELDERS IN SOUTH KOREA

${ }^{1}$ Se Yeong Kim*, ${ }^{2}$ Dong Mug Kang, ${ }^{3}$ Jung II Kim. 'Busan Workers' Health Centre and Pusan National University, Pusan, Korea; ${ }^{2}$ Occupational and Environmental Medicine, Pusan National University Yangsan Hospital, Pusan, Korea; ${ }^{3}$ Occupational and Environmental Medicine, Dong- A University Hospital, Pusan, Korea

\subsection{6/oemed-2018-ICOHabstracts. 1309}

Introduction Workplace health monitoring using periodic spirometry has been recommended as a tool for prevention of respiratory disease. The Spirometry Longitudinal Data Analysis (SPIROLA) software is an integrated visual and quantitative tool to aid in monitoring lung function in individuals participating in spirometry-based health monitoring programs developed by Centres for Disease Control and Prevention (CDC).

Methods We analysed the annual lung function decline in 385 male workers of the Korean Shipyard Welder Cohorts by SPIROLA. To evaluate the annual decline of spirometry data, we analysed the longitudinal spirometry data collected from 2010 through the year 2015 in workers who had at least four follow-up tests. We estimated each person's rate of $\mathrm{FEV}_{1}$ and FVC decline and group's rate of $\mathrm{FEV}_{1}$ and FVC decline.

Result The results on 385 workers with 4 or more years of follow-up showed that the mean rate of $\mathrm{FEV}_{1}$ decline was higher than that for the Korean population. And decline of lung function in welders suggest a greater effect in smoking status.

Discussion This study indicates the need for respiratory disease prevention and intervention in this shipyard welders. The computerised annual lung function decline program is very useful of workers' respiratory health prevention. So We need to develop a program suitable for workplace environment in Korean.

\section{WORK-RELATED ASTHMA AMONGST ASTHMATIC PATIENTS IN THE EASY ASTHMA CLINICS IN KHON KAEN, THAILAND}

${ }^{1} \mathrm{~N}$ Chaiear* ${ }^{*}{ }^{1} \mathrm{~W}$ Jongkumchok, ${ }^{2} \mathrm{~W}$ Boonsawat, ${ }^{3} \mathrm{~S}$ Chaisuksant. ${ }^{1}$ Division of Occupational Medicine, Department of Community Medicine, Faculty of Medicine, Khon Kaen University Khon Kaen, Thailand; ' 2 Department of Medicine, Faculty of Medicine, Khon Kaen University, Khon Kaen, Thailand; ${ }^{3}$ Department of Medicine, Khon Kaen Hospital, Khon Kaen, Thailand

\subsection{6/oemed-2018-ICOHabstracts. 1310}

Introduction Work-related asthma (WRA) amongst asthmatic patients in worldwide country are about 15\%-25\%. In Thailand, there are at least 3 million asthmatics patients but there have never been a figure of WRA presented. This is the first study in Thailand where a proportion of WRA amongst asthmatic patients was figured.

Methods A descriptive study was performed. The samples of 523 asthmatic patients in the Easy Asthma Clinics were 